\section{Stressing out over miRNA}

\section{By Tim Fulmer, Senior Writer}

Two papers, one published in the Proceedings of the National Academy of Sciences ${ }^{1}$ and the other in Nature Immunology, ${ }^{2}$ identify several microRNAs that play a role in cellular stress triggered by myocardial infarction or viral infection and tumorigenesis. Although both papers provide potential new targets, hurdles such as proper therapeutic design and delivery remain to be cleared.

The studies illustrate two different methods by which miRNAs can regulate cellular processes. The research in PNAS shows how a single miRNA regulates the expression of a family of functionally related proteins, whereas the Nature Immunology research shows how multiple miRNAs regulate expression of two functionally related genes.

miRNAs are endogenous 23-nucleotide single-stranded RNAs that control gene expression post-transcriptionally by binding to particular sequences in the untranslated regions of target mRNA molecules. This process either inhibits protein translation or promotes mRNA degradation. Individual miRNAs can simultaneously target multiple mRNAs and thus potentially repress the expression of entire groups or functional networks of proteins (see Box 1, "The far-reaching effects of microRNAs").

"For both labs, key next steps would include evaluating the efficacy of miRNA antagonists or mimics in the appropriate preclinical disease models, as well as determining appropriate strategies for local or systemic delivery," said David Brown, director of R\&D at Asuragen Inc., which is working on miRNA cancer therapeutics through its wholly owned Mirna Therapeutics Inc. subsidiary.

\section{Flustering fibrosis}

Researchers at the University of Texas Southwestern Medical Center (UT Southwestern) and miRagen Therapeutics Inc., led by Eric Olson, professor of molecular biology at UT Southwestern and cofounder of miRagen, have identified miRNA-29 as a potential master regulator of fibrosis.

In their PNAS paper, the authors sought to identify miRNAs involved in cardiac remodeling following myocardial infarction (MI; see Figure 1, "Modulating microRNA to minimize stress"). The researchers built on their previous work that identified multiple miRNAs in cardiac hypertrophy and heart failure. ${ }^{3}$ These included miRNA-208, currently one of miRagen's lead preclinical targets for heart failure.
"Our paper illustrates a general strategy for validating potential miRNA targets," said William Marshall, president and CEO of miRagen. "We begin with established animal models of a particular disease class, in this case cardiac disorders. Next, we look for miRNAs that are consistently dysregulated across these models compared with healthy animals. Finally, we correlate these dysregulated miRNAs with levels of the corresponding miRNA in diseased human tissue."

In mouse models of left coronary artery occlusion, multiple miRNAs were dysregulated 3 and 14 days post-MI in the border zone of the infarct region compared with the same miRNAs in hearts of healthy mice. Several of these miRNAs were similarly dysregulated in cardiac tissue isolated from MI patients receiving a heart transplant. In particular, miRNA-29 was substantially downregulated in post-MI human heart samples compared with what was seen in cardiac tissue from patients with nonfailing hearts $(p<0.05)$.

Using a computational approach, the authors subsequently predicted that miRNA-29 would target a number of mRNAs that encode proteins implicated in cardiac fibrosis, including collagen I, collagen III, elastin and fibrillin. Indeed, analysis of cardiac tissue from the same MI mouse models revealed that lower miRNA-29 expression was significantly correlated with higher expression of collagens and fibrillin $(p<0.05)$.

In healthy mice, tail vein injection of an anti-miRNA-29 oligonucleotide resulted in higher collagen expression in the heart and liver after three weeks than did injection of saline or mock control oligonucleotide. Conversely, in cultured murine cardiac fibroblasts, an miRNA-29 mimic lowered collagen levels compared with those seen in untreated control fibroblasts.

"Our results suggest that the downregulation of miR-29 contributes to cardiac fibrosis and that strategies to maintain miR-29 expression may be beneficial in the settings of fibrotic diseases," the authors concluded.

"The study shows that miRNA-29 targets multiple mRNAs that code for proteins implicated in fibrosis-in particular, collagens I and III," Olson told SciBX. "Thus, miRNA-29 may very well be a master regulator of fibrosis not only in the heart but perhaps also in other organs like the liver."

\section{Heart start}

There are no approved disease-modifying therapies for conditions that involve fibrosis of the heart or liver. Instead, treatments are palliative and essentially serve to delay the potential need for an organ transplant. Although miRagen would not disclose its specific development plans for miRNA-29, Olson did say the company will continue to study the compound in cardiac fibrosis and, potentially, in related indications such as liver fibrosis.

"Next steps include evaluating the use of an miRNA-29 oligonucleotide mimic to elevate miRNA-29 levels in post-MI mouse hearts and thereby reduce levels of downstream proteins that contribute to cardiac fibrosis," said Eva van Rooij, first author on the PNAS paper and postdoctoral researcher in Olson's lab at UT Southwestern. 
"A second potential strategy for slowing down or preventing fibrosis would be to treat post-MI hearts with compounds that activate miRNA-29 transcription, thus increasing cellular levels of miRNA-29 and potentially reducing levels of their target mRNAs," added Olson.

Peter Linsley, CSO of Regulus Therapeutics LLC, agreed that key next steps should include administering miRNA-29 mimics to animal models of cardiac disease.

"While delivery strategies of single-stranded antisense antagomir technology have been worked out reasonably well over the years, delivering double-stranded miRNA mimics will likely present its own challenges," he noted. "Thus, studies of miRNA mimics in the appropriate preclinical disease models will be an important next step for better understanding the potential therapeutic applicability of these compounds in humans."

Regulus is a joint venture between Alnylam Pharmaceuticals Inc. and Isis Pharmaceuticals Inc. The company's lead compound, a therapeutic that antagonizes host miRNA-122, is in preclinical testing to treat or prevent $\mathrm{HCV}$ infection. The compound has shown in vivo efficacy in mice. ${ }^{4}$

Olson noted that "one of the advantages of designing miRNA thera- peutics in cardiac disorders is that highly invasive methodologies are typically the standard of care in cardiology. This gives us the option of using catheter-based techniques for direct local delivery of an miRNA mimic or antagonist into cardiac tissue. Hence, off-target effects in noncardiac tissue and reduced efficacy from oligonucleotide degradation in the plasma are potentially avoided."

He added, "Nevertheless, over the much longer term we are interested in exploring systemic delivery strategies for miRNA therapeutics in those indications where highly invasive methods may have little patient or doctor compliance."

miRagen has rights to IP covering miRNA-29 antagonists as well as mimics. Indeed, blocking the molecule to upregulate collagen production actually could be beneficial in nonfibrotic diseases.

"One can envision targeting miRNA-29 in the context of wound healing, where inducing collagen expression would be a desirable outcome," said Olson.

\section{Stress cases}

Stress-related miRNAs could also offer new avenues for cancer therapy. In the Nature Immunology paper, Ofer Mandelboim and colleagues

\section{Box 1. The far-reaching effects of microRNAs.}

Two papers recently published in Nature illustrate the subtle regulatory effects exerted by microRNAs on the proteome, the entire complement of proteins expressed by an organism's genome..$^{9,10}$

In the first article, by David Bartel and colleagues at Massachusetts Institute of Technology's Whitehead Institute and Harvard University, the researchers used a quantitative mass spectrometric approach, called stable isotope labeling with amino acids in cell culture (SILAC), to study the global impact on protein output of either ectopically expressing an miRNA (miRNA-124, miRNA-1 or miRNA-181) in human cervical cancerderived HeLa cells, or of knocking out a gene encoding a single miRNA (miRNA-223) in mouse neutrophils.

In the second study, Nikolaus Rajewsky and colleagues at the Max Delbrück Center for Molecular Medicine and the University of Glasgow performed similar experiments. They ectopically overexpressed miRNAs (miRNA-1, miRNA-16, miRNA$30 a$, miRNA-155 or let-7b) in HeLa cells and, in the case of let- $7 \mathrm{~b}$, contrasted those results with the knockdown, also in HeLa cells, of the let-7b miRNA.

In this second paper, protein levels were assessed using a variation called pulsed SILAC, which allowed the researchers to determine changes in the proteome within a defined and short window of time.

Both studies showed for the first time that overexpression or knockout of a single miRNA results in widespread but modest changes in the expression of most cellular proteins. In addition, both sets of results showed that the levels of a particular protein in the cell were the result of a fine balance between inhibition of protein translation and enhanced mRNA degradation, two processes regulated by the corresponding miRNA-mRNA interaction.

"miRNAs provide a layer of regulatory control over the proteome that is intermediate between transcriptional control at the gene level and posttranslational control at the protein level," Bartel told SciBX. "Of course, all of these layers acting in concert will influence the phenotype of a particular cell. However, one of the attractive aspects of the miRNA regulatory layer is its structural basis in complementary base-pairing interactions between miRNAs and mRNAs. These interactions will probably be easier to identify, characterize and potentially target than the protein-DNA and protein-protein interactions that characterize the other regulatory layers."

The extent to which one miRNA can modulate protein levels globally has implications for the specificity and thus the design of miRNA-targeting therapeutics. Although the levels of most proteins analyzed by Bartel and Rajewsky's teams were somewhat influenced by the dysregulation of a single miRNA, strong effects on specific mRNAs and proteins were also seen.

For example, in Bartel's miRNA-223 knockdown experiments, the list of the most strongly derepressed proteins included cathepsin L, insulin-like growth factor-1 receptor and cathepsin Z. Increased levels of these three proteins may explain the previously observed proinflammatory phenotype linked with miRNA-223 mutant mice. ${ }^{11}$

"Based on what we've seen in our studies, it's likely that dysregulation of any highly conserved miRNA will result in many proteins having measurably altered levels," said Bartel. "However, only a few of these proteins will represent vulnerable nodes within a regulatory network - these could represent the most attractive therapeutic targets."

- Gaspar Taroncher-Oldenburg, Executive Editor 


\section{COVER STORY}

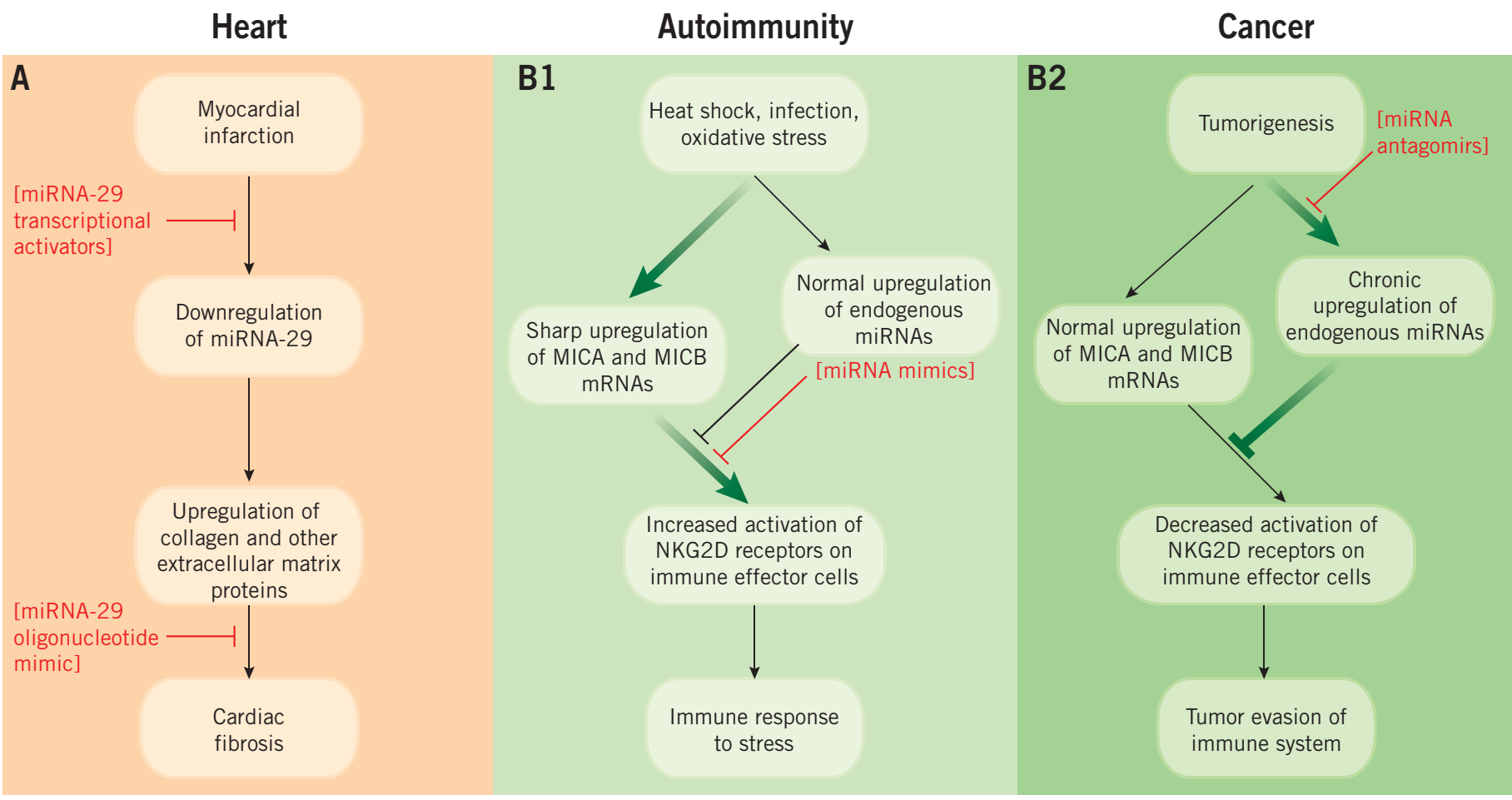

Figure 1. Modulating microRNA to minimize stress. Papers published separately in Proceedings of the National Academy of Sciences and Nature Immunology spotlight the central role microRNAs play in modulating the cellular response to a variety of stresses, including metabolic stress, viral infection and tumorigenesis.

(A) In mouse models of myocardial infarction (MI), researchers at miRagen Therapeutics Inc. and University of Texas Southwestern Medical Center found that levels of miRNA-29 were downregulated in response to post-MI stress. Lower miRNA-29 levels correlated with the upregulation of several extracellular matrix proteins previously implicated in cardiac remodeling and fibrosis, including collagen. Thus the researchers suggest that strategies to increase miRNA-29 levels might help slow or prevent progression to fibrosis.

(B1) Researchers at The Hebrew University showed that cellular miRNAs regulated expression of ligands MICA and MICB that activate natural killer (NK) cells under a variety of conditions.

Under conditions of cellular stress, expression of major histocompatibility class I polypeptide-related sequence A (MICA) and MICB mRNA increases to levels that prevent suppression by miRNAs and thus allows activation of downstream immune effector cells to attack the stressor. Under normal conditions, the miRNAs block expression of MICA and MICB mRNAs to ensure that downstream immune effector cells remain inactive and do contribute to a potential autoimmune reaction.

Thus the researchers suggest miRNA mimics could be useful for enhancing suppression of NK cell activation where levels of endogenous miRNAs are not sufficient.

(B2) In tumors, some of the same miRNAs are chronically overexpressed, resulting in excessive downregulation of NK cells and consequent evasion of immune cell recognition by tumors. To counter this effect, the researchers suggested that strategies to antagonize the overexpressed miRNAs might have antitumor activity.

at The Hebrew University studied the analogous role of miRNAs in virus- and cancer-induced stress. Mandelboim is professor of general and tumor immunology at the university.

The team's previous work had shown how a human cytomegalovirus (HCMV) miRNA downregulated expression of the natural killer (NK) cell ligand major histocompatibility class I polypeptide-related sequence $\mathrm{B}$ (MICB). This resulted in lower killing potential of NK cells during infection and thus evasion of the host immune system by the virus. ${ }^{5}$

MICB and a related molecule, MICA, are ligands of the natural killer group 2, member D receptor (NKG2D receptor) on the surface of NK cells. Activation of the NK2GD receptor by its ligands occurs when host cells are subjected to stress such as heat shock, infection and tumorigenesis. ${ }^{6}$

In the Nature Immunology paper, the researchers set out to identify endogenous human miRNAs that target MICA and MICB mRNAs. The researchers found several miRNAs that were expressed in multiple human tissues. Under normal conditions, these miRNAs prevent overexpression of MICA and MICB and subsequent activation of effector cells that could potentially target self cells (see Figure 1, "Modulating microRNA to minimize stress"). 
However, under conditions of short-term stress such as heat shock or viral infection, MICA and MICB mRNA in cultured cells increased to levels that were so high they could not be downregulated by miRNA. The authors suggested that the higher levels of mRNA could be saturating the suppressive capacity of the miRNAs, resulting in downstream activation of immune effector cells in response to stress.

Conversely, under the chronic condition of tumorigenesis, sustained overexpression of miRNAs resulted in excessive downregulation of MICA and MICB, which led to less activation of immune effector cells expressing NKG2D receptors and consequently enabled tumors to evade host immune recognition.

"As MICA and MICB are expressed in a wide variety of hazardous conditions, such as autoimmunity, cancer and viral infections, manipulating the expression of their cellular miRNAs might have considerable therapeutic value," the authors concluded.

According to Mandelboim, next steps include identifying endogenous miRNAs that regulate mRNAs targeted by other members of the herpes virus family, including Epstein-Barr virus, Kaposi's sarcoma-associated herpesvirus (KSHV) and herpes simplex.

Mandelboim told SciBX that Hebrew University has filed multiple patent applications for the use of multiple miRNA molecules and antisense oligonucleotides in cancer and other indications.

Mirna Therapeutics is pursuing a somewhat different approach from that followed by Mandelboim.

According to Asuragen president Rollie Carlson, "First, we identify miRNAs that are dysregulated in cancerous human tissue and then characterize the targets and function of these miRNAs in vitro. This step allows us to confirm that a given miRNA plays a causal role at the cellular level of a particular cancer."

Once causality has been shown, he said, "we can next design the appropriate therapeutic, an antagonist or mimic, as the case may be, which can be tested for efficacy in a number of different animal models of the cancer. Finally, we could then evaluate the appropriate technologies for delivering the therapeutic specifically to the tumor."

Mirna's lead compound is a let-7 miRNA mimic that has shown antitumor efficacy in a rodent lung cancer xenograft model when delivered intranasally. ${ }^{7}$ Carlson told SciBX that the company plans to carry out toxicity studies in primates or dogs and hopes to submit an IND in two to three years.

In a move showing the degree to which the miRNA space is heating up, Carlson indicated that "Asuragen is currently in discussions with a large VC group to substantially increase funding for Mirna Therapeutics," which would reduce Asuragen's ownership stake.

This would add to the recently announced deals in this space giving Alnylam Pharmaceuticals IP rights over a broad range of technologies related to RNA-induced gene activation (RNAa), including miRNA-based applications of RNAa. ${ }^{8}$

On its own, Asuragen develops and markets miRNA-based diagnostic tools for the early detection of cancer.

\section{REFERENCES}

1. van Rooij, E. et al. Proc. Natl. Acad. Sci. USA; published online Aug. 4, 2008; doi:10.1073/pnas.0805038105

Contact: Eric Olson, University of Texas Southwestern Medical Center, Dallas, Texas e-mail: eric.olson@utsouthwestern.edu

2. Stern-Ginossar, N. et al. Nat. Immunol.; published online Aug. 1, 2008; doi:10.1038/ni.1642

Contact: Ofer Mandelboim, The Hebrew University, Jerusalem, Israel e-mail: oferm@ekmd.huji.ac.il

3. van Rooij, E. \& Olson, E. J. Clin. Invest. 117, 2369-2376 (2007)

4. Krutzfeldt, J. et al. Nature 438, 685-689 (2005)

5. Horwaitz, E. et al. Science 317, 376-381 (2007)

6. Eagle, R. \& Trowsdale, J. Nat. Rev. Immunol. 7, 737-744 (2007)

7. Esquela-Kerscher, A. et al. Cell Cycle 7, 1-6 (2008)

8. Osherovich, L. SciBX 1(28), 1-4; Aug. 14, 2008

9. Baek, D. et al. Nature; published online July 30, 2008; doi:10.1038/nature07242

Contact: David Bartel, Whitehead Institute, Massachusetts Institute of Technology, Cambridge, Mass. e-mail: dbartel@wi.mit.edu

10. Selbach, M. et al. Nature; published online July 30, 2008; doi:10.1038/nature07228

Contact: Nikolaus Rajewsky, Max Delbrück Center for Molecular Medicine, Berlin, Germany e-mail: nikolaus.rajewsky@mdc-berlin.de

11. Johnnidis, J. et al. Nature; published online Feb. 17, 2008; doi:10.1038/nature06607

\section{COMPANIES AND INSTITUTIONS MENTIONED}

Alnylam Pharmaceuticals Inc. (NASDAQ:ALNY), Cambridge, Mass. Asuragen Inc., Austin, Texas

Harvard University, Cambridge, Mass.

The Hebrew University, Jerusalem, Israel

Isis Pharmaceuticals Inc. (NASDAQ:ISIS), Carlsbad, Calif.

Massachusetts Institute of Technology, Cambridge, Mass.

Max Delbrück Center for Molecular Medicine, Berlin, Germany

miRagen Therapeutics Inc., Boulder, Colo.

Mirna Therapeutics Inc., Austin, Texas

Regulus Therapeutics LLC, Carlsbad, Calif.

University of Glasgow, Glasgow, U.K.

University of Texas Southwestern Medical Center, Dallas, Texas Whitehead Institute, Cambridge, Mass. 